\title{
Transport by Molecular Motors in the Presence of Static Defects
}

\author{
Yan Chai · Reinhard Lipowsky · Stefan Klumpp
}

Received: 25 September 2008 / Accepted: 28 February 2009 / Published online: 25 March 2009

(c) The Author(s) 2009. This article is published with open access at Springerlink.com

\begin{abstract}
The transport by molecular motors along cytoskeletal filaments is studied theoretically in the presence of static defects. The movements of single motors are described as biased random walks along the filament as well as binding to and unbinding from the filament. Three basic types of defects are distinguished, which differ from normal filament sites only in one of the motors' transition probabilities. Both stepping defects with a reduced probability for forward steps and unbinding defects with an increased probability for motor unbinding strongly reduce the velocities and the run lengths of the motors with increasing defect density. For transport by single motors, binding defects with a reduced probability for motor binding have a relatively small effect on the transport properties. For cargo transport by motors teams, binding defects also change the effective unbinding rate of the cargo particles and are expected to have a stronger effect.
\end{abstract}

Keywords Molecular motors $\cdot$ Defective filaments $\cdot$ Motor walks $\cdot$ Motor traffic $\cdot$ Run length $\cdot$ Lattice models

\section{Introduction}

The interior of living cells is characterized by highly organized complex structures. To build and maintain these internal structures, cells rely on directed active transport of various types of cargoes to different destinations within the cell. This transport is driven by molecular motors which use the energy derived from the hydrolysis of adenosine triphosphate (ATP)

Y. Chai · R. Lipowsky

Max Planck Institute of Colloids and Interfaces, Science Park Golm, 14424, Potsdam, Germany

Y. Chai

e-mail: chai@mpikg.mpg.de

S. Klumpp (ه)

Center for Theoretical Biological Physics and Department of Physics, University of California at San Diego, La Jolla, CA 92093-0374, USA

e-mail: klumpp@ctbp.ucsd.edu 
to move along cytoskeletal filaments $[14,44]$. There are three large families of cytoskeletal motors, kinesins and dyneins, which move along microtubules, and myosins, which move along actin filaments [14, 44].

Since cells provide crowded environments, motors moving along filaments encounter a variety of other molecules bound to the same filaments, which may hinder their movement. These obstacles may represent other motors of the same type, and the traffic phenomena that arise in such systems with many motors have been studied extensively in recent years. Many theoretical studies have explored the formation of traffic jams and non-equilibrium phase transitions [8, 16, 17, 31, 38, 39], and traffic jams of molecular motors have recently been observed in several experimental studies [25, 29, 38]. A system with two different species of motors that move into opposite direction has also been studied theoretically and is predicted to exhibit spontaneous symmetry breaking and the formation of separate traffic lanes for the two directions [19].

In addition to molecular motors, a variety of other molecules can bind to filaments and affect the movement of these motors. An important example is given by microtubuleassociated proteins (MAPs), which bind to microtubules to control their structure and stability. In addition, MAPs can modulate the movement of the motors along the microtubules. When overexpressed in vivo $[2,7,43]$ or added to microtubule gliding assays with kinesin or dynein motors in vitro $[11,12,32,40]$, MAPs decrease or completely inhibit motility of motors. More recent experiments using lower concentrations of MAPs and tracking the movements of individual motors show that most MAPs studied so far affect motor movements by modulating binding of the motors to microtubules. For example, the tau protein, a MAP specific for neurons, has been shown to decrease the binding of kinesin and dynein motors to microtubules [5, 48, 52]. Its effect depends on the tau isoform [52], is more pronounced for kinesin than for dynein motors [5, 7, 53], and has a stronger effect on cargoes pulled by several motors than on individual motors, see $[21,48,52]$ and the discussion below. These subtle and highly specific effects seen at low tau concentration [5, 48, 52] suggest that tau (and other MAPs) may play important roles as regulators of transport in cells, and function as general transport inhibitors only under pathological conditions [33]. For example, the differential effects on kinesins and dynein suggest that tau can control the direction of motion of cargoes that are carried by both types of motors, as discussed in [36].

When modeling large-scale transport by molecular motors, static molecules bound to the filaments can be considered as local properties of the filament. They represent static or quenched defects of the filament that affect the motor dynamics locally. The same theoretical description may then be used for other types of defects that cause local effects on motor transport. Such defects may for example be local modifications of the filament themselves such as microtubule lattice defects or a variety of post-transcriptional modifications of tubulin, the subunit of microtubules. Some of these modifications have been shown to affect the microtubule-binding or the movement of motors [27, 28, 42]. Finally, in addition to these naturally occurring defects, artificial 'roadblocks' such as inactive motor mutants have been used in several experiments to perturb the movement of active motors in order to study the mechanisms of motor function [3, 47].

In this paper, we study the effects of various types of defects on the movements of molecular motors using the lattice model introduced in [31]. Here we use the simple description of the dynamics of motor stepping provided by the lattice model to distinguish three basic types of static defects and to study their effects on single motors as well as on the motor traffic in many-motor systems. The three basic types of defects are given by filament sites that differ from the other filament sites in one of three motor parameters: (i) stepping defects have an altered forward stepping probability, (ii) unbinding defects have an altered unbinding probability, and (iii) binding defects have an altered binding probability. Some cases that 
have been studied previously can be considered as special cases of this general approach. For example a single stepping defect has been studied in [41], and a single unbinding defect without unbinding from non-defect sites in [34]. Very recently, binding defects have been studied in [10]. Stepping defects have also been investigated extensively for one-dimensional exclusion processes $[15,18,24,50]$, which, in our model, correspond to movement of motors along a filament without binding and unbinding. We also note that in the statistical mechanics literature such defects are classified as 'sitewise' disorder, since the anomalous properties are related to a fraction of the lattice sites, as opposed to the case of 'particlewise' disorder, for which some of the moving particles exhibit anomalous properties [26].

The paper is organized as follows: In Sect. 2, we introduce the lattice model and the system geometry used in this study as well as the description and classification of defects. We discuss the modeling of known biological defects such as MAPs within this model. We then study stepping defects in Sect. 3, unbinding defects in Sect. 4 and binding defects in Sect. 5. We conclude with a few general remarks on the use of defects in transport.

\section{Lattice Model with Different Types of Defects}

\subsection{Lattice Model for the Traffic of Molecular Motors}

To study the effects of various types of defects and the transport by molecular motors, we extend the lattice model introduced in [31], which we have previously used to describe both the movement of single motors [20, 31, 37] as well as the traffic in many-motor systems $[17,31]$. This model describes the movements of a single molecular motor along a filament as a random walk on a (generally three-dimensional) lattice, which contains one or several lines of lattice sites that represent filaments. The lattice constant $\ell$ is given by the step size of a motor moving actively along a filament. Per unit time $\tau$, a motor at a filament site makes a forward step along the filament with probability $\alpha$, unbinds to each of the four neighboring non-filament sites with probability $\epsilon / 6$, and remains at the same site with probability $\gamma=1-\alpha-4 \epsilon / 6$. Motors at non-filament sites perform symmetric random walks and move to each nearest neighbor site with probability $1 / 6$. The choice of this probability implies that the time scale $\tau$ is given by the diffusion coefficient of unbound motors $D_{\mathrm{ub}}$ as $\tau=\ell^{2} / D_{\mathrm{ub}}$. If an unbound motor moves to a filament site, it binds to it with the sticking probability $\pi_{\mathrm{ad}}$. If $\pi_{\mathrm{ad}} \neq 1$, this condition modifies the probability for the movement from a non-filament site to a filament site to $\pi_{\text {ad }} / 6$. In general, we can model both freely suspended filaments, for which the filament site is connected to four neighboring non-filament sites, and immobilized filaments, for which the number of nearest neighbors is at most equal to three. In the simulations reported below, we focused on freely suspended filaments.

In addition to the dynamics of single motors, the lattice model can also describe systems with many interacting motors. In the simplest case, these motors interact only through their mutual exclusion from lattice sites, which is implemented in the model by not allowing any steps to sites that are occupied by another motor. Typically the density of motors at nonfilament sites is much lower than at filament sites, so that the exclusion rule affects mainly binding to the filament and movement along it. By virtue of this exclusion rule, our model is a variant of driven lattice gas models or exclusion processes, which have been studied extensively as model systems for transport processes and non-equilibrium phase transitions $[45,46]$.

Throughout this article, we will study systems that have a tube-like geometry as shown in Fig. 1. In these systems a single filament is located on the axis of a cylindrical tube 


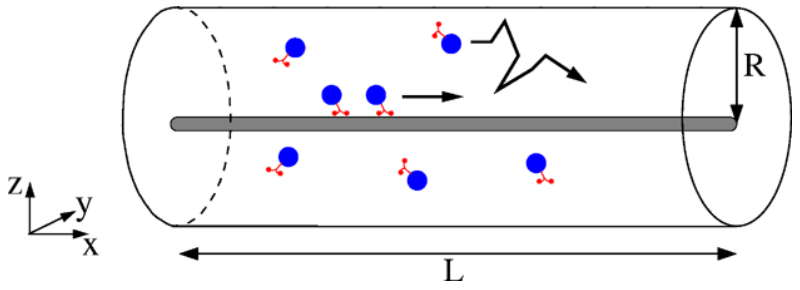

Fig. 1 Molecular motors inside a cylindrical tube with a filament aligned along its axis. This tube system mimics the geometry of elongated cellular structures such as axons. The tube has the length $L$ and the radius $R$. Motors bound to the filament move actively along the filament in a directed fashion, while unbound motors perform diffusive movements. The boundary condition is periodic along the $x$-axis

with length $L$ and radius $R$. This geometry mimics the structure of some types of cells, such as axons of nerve cells or hyphae of fungi, which are approximately tubular and have a unidirectional microtubule cytoskeleton [9]. Similar tube-like systems have previously been studied with various types of boundary conditions [17, 22, 31, 35]. In order to keep the discussion simple, we will focus on periodic boundary conditions in the following.

For periodic boundary conditions, the case without defects is particularly simple and has been solved exactly [17]. In this case, the densities of bound and unbound motors, $\rho_{\mathrm{b}}$ and $\rho_{\mathrm{ub}}$, respectively, are spatially homogeneous and satisfy a binding-unbinding balance condition,

$$
\pi_{\mathrm{ad}} \rho_{\mathrm{ub}}\left(1-\rho_{\mathrm{b}}\right)=\epsilon \rho_{\mathrm{b}}\left(1-\rho_{\mathrm{ub}}\right) \approx \epsilon \rho_{\mathrm{b}},
$$

and the motor current $J$ along the filament is given by $J=\alpha \rho_{\mathrm{b}}\left(1-\rho_{\mathrm{b}}\right)$. In the case of a single motor, the balance equation is

$$
\pi_{\mathrm{ad}} \rho_{\mathrm{ub}}=\epsilon \rho_{\mathrm{b}},
$$

from which one can derive the steady-state probability that the motor is bound to the filament, $P_{\mathrm{b}}=\sum_{x} \rho_{\mathrm{b}}=\rho_{\mathrm{b}} L / \ell$, which is given by

$$
P_{\mathrm{b}}=\frac{\pi_{\mathrm{ad}}}{\pi_{\mathrm{ad}}+\epsilon N_{\mathrm{ch}}}
$$

where $N_{\mathrm{ch}}$ is the number of unbound channels, i.e., the number of lines of lattice sites parallel to the filament in a discretized tube with cross section $\phi=\left(1+N_{\mathrm{ch}}\right) \approx \pi R^{2}$ for sufficiently large radius $R$. The effective motor velocity, averaged over the bound and unbound states of the motor, is then obtained as

$$
v_{\mathrm{eff}}=P_{\mathrm{b}} v_{\mathrm{b}}=\frac{\alpha \pi_{\mathrm{ad}}}{\pi_{\mathrm{ad}}+\epsilon N_{\mathrm{ch}}} \frac{\ell}{\tau}
$$

where $v_{\mathrm{b}}=\alpha \ell / \tau$ is the velocity of the bound motor.

\subsection{Lattice Model with Different Types of Defects}

Inhomogeneities of the filament such as those mentioned in the introduction may affect one or several of the motor properties. This can be described within the lattice model by modifying one or several of the hopping probabilities compared to the homogeneous situation. In 
Fig. 2 Three types of defects studied in this article:

(a) Stepping defect indicated by an encircled site with a modified forward stepping probability $\alpha_{\text {def }}$ compared to other filament sites.

(b) Unbinding defect with a modified unbinding probability

$\epsilon_{\text {def }}$. (c) Binding defect with a modified binding probability

$\pi_{\text {def }}$. All other transition probabilities are the same at the defect site as at the other filament sites

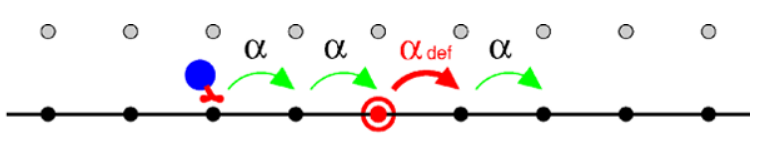

(a)

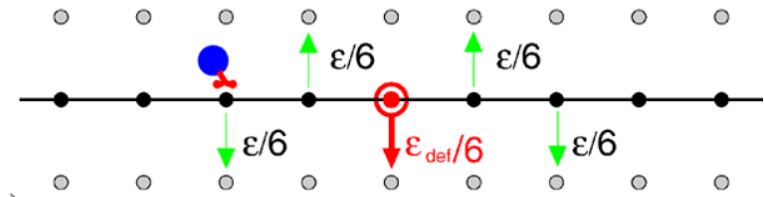

(b)

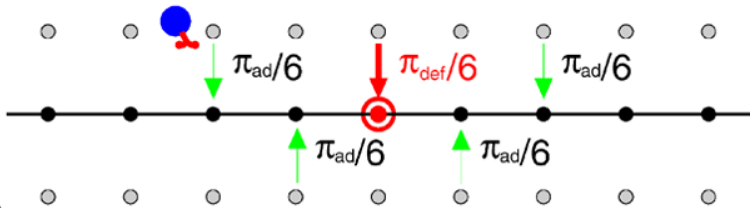

(c)

the following, we distinguish three basic types of defects which are characterized by a single parameter that differs from the homogeneous case as shown in Fig. 2: (i) Stepping defects have a changed probability $\alpha_{\text {def }}$ for forward movement, but unchanged binding probability $\pi_{\mathrm{ad}}$ and unbinding probability $\epsilon$; (ii) unbinding defects have a changed unbinding probability $\epsilon_{\text {def }}$; and (iii) binding defects have a modified sticking probability $\pi_{\text {def }}$. In all three cases, the dwell probability $\gamma_{\text {def }}$ also needs to be adapted, so that the sum of all probabilities is again equal to one.

More complicated types of defects can be considered as combinations of these basic defects. For example an inaccessible site due to a large immobile protein bound to the filament, such as an inactive mutant motor, can be described by a combination of a stepping defect and a binding defect with $\alpha_{\text {def }}=\pi_{\text {def }}=0$.

Table 1 lists examples of defects that have been characterized experimentally and summarizes their effects on molecular motors. Microtubule-associated proteins (MAPs) such as the tau proteins essentially represent binding defects (an exception is MAP4 [49], see Table 1). They reduce the binding rate of kinesin to microtubules and have no or only a weak effect on the velocity of bound kinesins as well as on the unbinding rate or the run length, the distance moved along the filament before unbinding [48, 52]. Similar effects have been observed for dynein motors [5], which in general are less affected by MAPs than kinesins. In vivo, tau has also been shown to reduce the run length for vesicular cargoes. The increase in unbinding rate for these cargoes is most likely a consequence of the fact that these cargoes are pulled by several motors rather than a single motor, since for cargoes pulled by several motors, the unbinding rate is a function of the single-motor binding rate [21, 48]. This effect has been demonstrated in vitro for tau and beads pulled by several kinesins [52]. Therefore defects that are binding defects for individual motors, can be both binding and unbinding defects for cargoes pulled by multiple motors.

The effects of post-translational modifications of microtubules on motor movements have not been characterized in much mechanistic detail. One case for which the effect is known is acetylation/deacetylation of a particular lysine residue of $\alpha$-tubulin for which it has been 
Table 1 Overview of different types of defects and their effects on molecular motor movement

\begin{tabular}{lllllll}
\hline Defects & Motors & Stepping & Unbinding & Binding & References & Note \\
\hline Tau & kinesin & - & - & reduced & {$[5,48,52]$} & a \\
Tau & kinesin & reduced & increased & reduced & {$[5]$} & $b$ \\
MAP2c & kinesin & - & - & reduced & {$[48]$} & \\
MAP4 & kinesin & reduced & - & - & {$[49]$} & $c$ \\
Tau & dynein-dynactin & reduced & increased & reduced & {$[5]$} & b \\
Tau & dynein & - & - & reduced & {$[53]$} & \\
De-acetylated $\alpha$-tubulin & kinesin & reduced & - & reduced & {$[42]$} & \\
Tubulin without E-hook & kinesin & reduced & - & - & {$[27]$} & \\
Inactive motor mutant & kinesin & reduced to 0 & $?$ & reduced to 0 & {$[3,47]$} & d \\
\hline
\end{tabular}

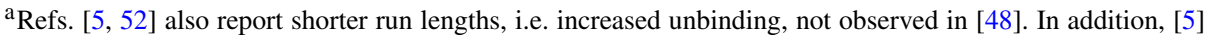
reports also a substantial fraction of immobile motors bound to microtubules

${ }^{b}$ Under the conditions of this experiment motors have rather long run lengths in the absence of tau, longer than in the experiments of $[48,52,53]$

${ }^{\mathrm{c}}$ The effect depends on which isoform of MAP4 is used. A 5-repeat isoform exhibits a strong effect, while the other isoforms studied showed only small effects [49]

${ }^{\mathrm{d}}$ Refs. [3, 47] report conflicting results for the effect on unbinding

shown that kinesin binds more strongly to the acetylated form than to the de-acetylated form [42]. Microtubules containing de-actelyated tubulin subunits therefore provide another example of binding defects. Microtubule lattice defects are believed to cause unbinding of motors, see, e.g. [4], and would thus represent unbinding defects. While this scenario is plausible, it has not been studied systematically and there is no direct experimental evidence for it.

Finally, the artificial 'roadblock' motor mutants used in [3, 47] represent blocked sites, i.e. combinations of a stepping defect with very low, essentially zero, stepping rate and a binding defect. Whether they also affect unbinding is unclear, since the two experiments in $[3,47]$ reported conflicting results, for a discussion see also [23]. In another recent experiment, some motors were inactivated by irreversible crosslinking to a microtubule to obtain blocked sites [6].

\section{Transport by Molecular Motors in the Presence of Stepping Defects}

\subsection{Single Motor with Stepping Defects}

We start by considering stepping defects. At a stepping defect, the motor has the forward stepping probability $\alpha_{\text {def }}$, while the binding and unbinding parameters are the same as at the other filament sites. We note that the probability to remain at the site is also changed compared to other sites and given by $\gamma_{\text {def }}=1-\alpha_{\text {def }}-4 \epsilon / 6$. We consider a single filament in a tube as shown in Fig. 1 with a density $\rho_{\text {def }}$ of stepping defect sites. To keep the discussion simple we study the case where the defect sites are arranged periodically on the filament. This situation is then equivalent to a system with a single defect, length $L=\ell / \rho_{\text {def }}$ and periodic boundary conditions.

First, we consider the effect of stepping defects on a single motor. As the stepping defects do not affect the binding and unbinding probabilities of the motor, one may expect 
that the binding probability is the same as in the absence of defects. However, relation (2), which describes a local balance of binding and unbinding, is not valid in the presence of stepping defects, since the motor densities are not constant along the filament because of the prolonged waiting times of the motor at the defect sites. Thus relation (2) has to be replaced by the global balance of binding and unbinding which remains valid if the densities are not constant and is given by

$$
\sum_{x} \pi_{\mathrm{ad}} \rho_{\mathrm{ub}}\left(x, y_{\mathrm{nn}}, z_{\mathrm{nn}}\right)=\sum_{x} \epsilon \rho_{\mathrm{b}}(x)
$$

where $y_{\mathrm{nn}}$ and $z_{\mathrm{nn}}$ are the perpendicular coordinates of a single channel of non-filament sites that are the nearest neighbors of the filament sites, e.g. $y_{\mathrm{nn}}=\ell$ and $z_{\mathrm{nn}}=0$. The inhomogeneity of the unbound density is relatively small because the fast motor diffusion tends to smooth the unbound density profile and taking the unbound density to be independent of the coordinates perpendicular to the filament, i.e. $\rho_{\mathrm{ub}}(x, y, z) \simeq \rho_{\mathrm{ub}}(x)$, is usually a very good approximation $[17,22]$. Within this "two-state" approximation, the probabilities $P_{\mathrm{b}}$ and $P_{\mathrm{ub}}$ to find the motors in a bound or an unbound state, respectively, are given by

$$
P_{\mathrm{b}}=\sum_{x} \rho_{\mathrm{b}}(x) \quad \text { and } \quad P_{\mathrm{ub}}=N_{\mathrm{ch}} \sum_{x} \rho_{\mathrm{ub}}(x),
$$

and satisfy the normalization condition

$$
P_{\mathrm{b}}+P_{\mathrm{ub}}=P_{\mathrm{b}}+N_{\mathrm{ch}} \sum_{x} \rho_{\mathrm{ub}}(x)=1 .
$$

The flux balance relation (5) then becomes

$$
\pi_{\mathrm{ad}} \frac{P_{\mathrm{ub}}}{N_{\mathrm{ch}}}=\pi_{\mathrm{ad}} \frac{1-P_{\mathrm{b}}}{N_{\mathrm{ch}}}=\epsilon P_{\mathrm{b}},
$$

which leads to

$$
P_{\mathrm{b}}=\frac{\pi_{\mathrm{ad}}}{\pi_{\mathrm{ad}}+\epsilon N_{\mathrm{ch}}},
$$

the same expression as for the case without stepping defects.

To obtain the effective velocity of the motor, we introduce an effective passing time to describe the movement of the bound motor. We assume that the motor spends the time $\tau_{0}=\tau / \alpha$ at a normal site and the time $\tau_{\text {def }}$ at a defect site. Since there are $L / \ell-1$ normal sites and only one defect site on a filament segment of length $L$, the total time to move through such segment is $t_{\text {tot }}=(L-\ell) \tau / \ell \alpha+\tau_{\text {def }}$, provided the motor typically remains bound to the filament during such a run. The velocity of a bound motor can be estimated by

$$
v_{\mathrm{b}, \mathrm{eff}}=\frac{L}{t_{\mathrm{tot}}}=\frac{\alpha}{1-\frac{\ell}{L}\left(1-\frac{\alpha \tau_{\mathrm{def}}}{\tau}\right)} \frac{\ell}{\tau} .
$$

The effective velocity, which characterizes the motor movement including the diffusive excursions upon unbinding, is then given by $v_{\mathrm{eff}}=v_{\mathrm{b}, \mathrm{eff}} P_{\mathrm{b}}$.

The time $\tau_{\text {def }}$ to pass a defect remains to be specified. In the limit of a sufficiently weak defect and sufficiently processive motors as assumed so far, this time is given by the inverse of the defect stepping probability, i.e. $\tau_{\text {def }}=\tau / \alpha_{\text {def }}$. In general, however, there are two ways in which a motor can pass a stepping defect: the motor can either slowly step through the 
defect along the filament or it may unbind from the filament and rebind to it after diffusing around the defect. The relative importance of these two pathways depends on their relative probabilities: when $\alpha_{\text {def }} \gg \epsilon$, the direct path through the defect dominates, while unbinding and diffusion will be the dominant pathway for $\alpha_{\text {def }} \ll \epsilon$.

If the stepping probability $\alpha_{\text {def }}$ at the defect is not large compared to the unbinding parameter $\epsilon$, the probability for the motor to take the diffusion channel is comparable with the probability to move forward along the filament. To estimate the contribution of the diffusion channel, we start with the limiting case $\alpha_{\text {def }}=0$, for which the motor can only take the diffusive channel to pass defect sites. We make the ansatz that the probability for taking the diffusive pathway is proportional to the unbinding probability $\epsilon$. For $\alpha_{\mathrm{def}}=0$, the time it takes the motor to pass the defect is then given by $\tau_{\text {def }}=\tau / q \epsilon$ and the effective motor velocity is given by

$$
v_{\mathrm{eff}} \simeq \frac{L}{t_{\mathrm{tot}}} P_{\mathrm{b}}=\frac{\alpha}{1-\frac{\ell}{L}+\frac{\ell}{L} \frac{\alpha}{q \epsilon}} \frac{\pi_{\mathrm{ad}}}{\pi_{\mathrm{ad}}+\epsilon N_{\mathrm{ch}}} \frac{\ell}{\tau} .
$$

In these expressions, $q$ is an unknown free parameter and should depend on the geometry of the system. For the parameters used in our simulations, we have determined this parameter by fitting the expression for $v_{\text {eff }}$ to the simulation data for $\alpha_{\text {def }}=0$, see Fig. 3(a), which leads to $q \simeq 0.25$.

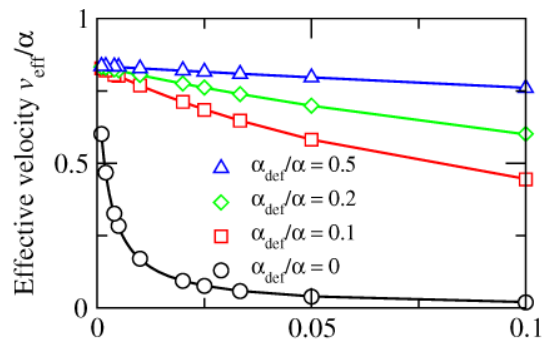

(a) Density of stepping defects $\rho_{\text {def }}$

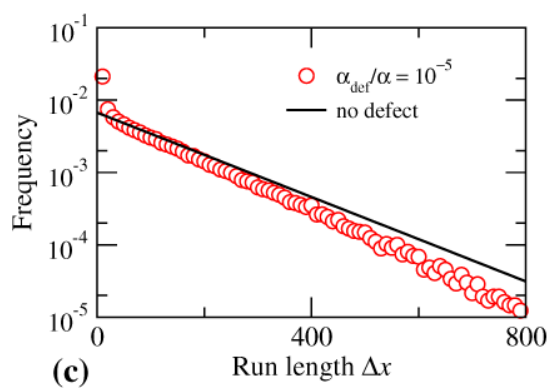

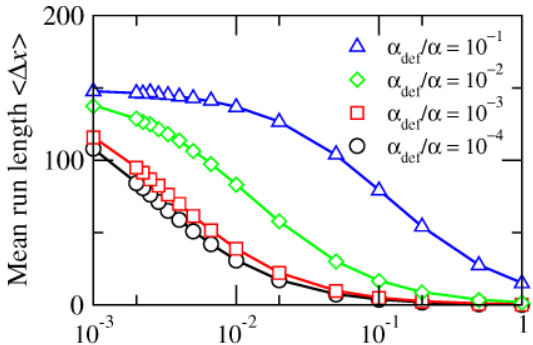

(b) Density of stepping defects $\rho_{\text {def }}$

Fig. 3 Transport properties of single motors in the presence of stepping defects: (a) Normalized effective velocity $v_{\text {eff }} / \alpha$ (in units of $\ell / \tau$ ) and (b) Average run length $\langle\Delta x\rangle$ (in units of $\ell$ ) as a function of the defect density $\rho_{\text {def }}$ for different values of the defect strength $\alpha_{\text {def }}$. The simulation data (symbols) are fitted by (13) and (15), respectively, with $q=0.25$ for all curves. (c) Run length histograms: without stepping defects (the straight line), the run length distribution is well fitted by a single exponential with average run length as given by (14); with stepping defects, the distribution has a peak for short run lengths and decays faster for large run lengths. The motor parameters are chosen to match the traffic of kinesins [17, 31]: $\alpha=0.0099333$, $\pi_{\mathrm{ad}}=1$, and $\epsilon=0.0001$. Furthermore we used $N_{\mathrm{ch}}=1960$ in (a) and (b), and $\rho_{\mathrm{def}}=0.001$ or $L / \ell=1000$ and $N_{\mathrm{ch}}=316$ in (c) 
For intermediate values of the stepping probability $\alpha_{\text {def }}$, both pathways contribute and the total probability to pass the defect is given by the sum of the probabilities for the two channels. The effective passing time $\tau_{\text {def }}$ for the motor is then proportional to $1 /\left(\alpha_{\mathrm{def}}+q \epsilon\right)$.

This expression implies that the effective bound motor velocity (or effective stepping rate) is given by

$$
v_{\mathrm{b}, \mathrm{eff}}=\frac{\alpha}{1-\frac{\ell}{L}+\frac{\ell}{L} \frac{\alpha}{\alpha_{\mathrm{def}}+q \epsilon}} \frac{\ell}{\tau} \equiv \alpha_{\mathrm{eff}} \frac{\ell}{\tau},
$$

and leads to the effective motor velocity

$$
v_{\mathrm{eff}}=\frac{\alpha}{1-\frac{\ell}{L}+\frac{\ell}{L} \frac{\alpha}{\alpha_{\mathrm{def}}+q \epsilon}} \frac{\pi_{\mathrm{ad}}}{\pi_{\mathrm{ad}}+\epsilon N_{\mathrm{ch}}} \frac{\ell}{\tau} .
$$

For the parameters used in Fig. 3(a), the free parameter $q$ has been determined by fitting this expression to the simulation data for $\alpha_{\text {def }}=0$ and applied to other cases of different value of $\alpha_{\text {def }}$. The curves obtained from expression (13) agree very well with the simulation data. We note however that this expression is not strictly valid in the limit of very weak defects with $\alpha_{\text {def }} \approx \alpha$. Putting $\alpha_{\text {def }}=\alpha$ in (12) leads to $\alpha_{\text {eff }}=\alpha+\mathcal{O}(\epsilon / \alpha)$, i.e. to a discrepancy of order $\epsilon / \alpha$, which is very small for processive motors.

Figure 3(a) shows that the velocity is reduced compared to the case without defects. As one might expect, this reduction is larger for stronger defects and/or for higher defect densities. If the defects are sufficiently strong, even very small defect densities lead to a substantial reduction of the velocity. For example, if 1 percent of sites on the filament are stepping defects with $\alpha_{\text {def }}=0$, the velocity of the single motor is reduced to about 20 percent compared to that without defects.

Another important property of the motors that is affected by stepping defects is their run length $\Delta x$, i.e. the distance a motor moves along a filament before unbinding from it. In the absence of defect sites on the filament, the average run length is given by

$$
\langle\Delta x\rangle_{0}=\frac{\alpha}{4 \epsilon / 6} \ell,
$$

and the distribution of run lengths decays exponentially as $\exp \left(-\Delta x /\langle\Delta x\rangle_{0}\right)$. The run length distribution for the case without defects is shown in Fig. 3(c), see the straight line. This exponential decay is modified by the presence of stepping defects, as also shown by the simulation data points in Fig. 3(c). In the presence of a low density of defects, the run length distribution decays slightly faster for large run length, and, in addition, develops a pronounced peak for short run length. This peak corresponds to short runs that start close to a defect and end at the same defect. Both effects lead to a reduction of the average run length. As the unbinding probability of the motors is not affected by stepping defects, the time a motor remains bound to a filament is the same with and without defects. ${ }^{1}$ However, the distance moved during this time is reduced if the motor encounters a defect. We can therefore estimate the average run length using the effective velocity of a bound motor, $v_{\mathrm{b}}$, which leads to

$$
\langle\Delta x\rangle=\frac{\alpha_{\text {eff }}}{4 \epsilon / 6} \ell=\frac{\langle\Delta x\rangle_{0}}{1-\frac{\ell}{L}+\frac{\ell}{L} \frac{\alpha}{\alpha_{\mathrm{def}}+q \epsilon}} .
$$

\footnotetext{
${ }^{1}$ We note that this is not necessarily the case if stepping and unbinding are coupled, e.g. if unbinding occurs during the step and is characterized by an unbinding probability per step rather than an unbinding rate. This scenario may arise for certain types of internal dynamics of the motors, see [23].
} 
The dependence of the mean run length on the density and the strength of the defects is shown in Fig. 3(b). For strong defects with $\alpha_{\text {def }} \ll \epsilon$, the precise value of $\alpha_{\text {def }}$ is irrelevant, as motors at the defect site typically unbind, before passing through the defect. For weaker defects, i.e. larger $\alpha_{\text {def }}$, the reduction of the run length is shifted towards larger defect densities. For $\alpha_{\text {def }}>\epsilon$, an approximately two-fold reduction of the run length is obtained when the defect density $\rho_{\text {def }}$ and $\alpha_{\text {def }} / \alpha$ have the same order of magnitude.

\subsection{Many Motors with Stepping Defects}

We now consider the effect of stepping defects on the traffic of many motors, which interact through mutual exclusion from filament sites. For the traffic of many motors in a tube with length $L$, we are interested in the following quantities [30]: (i) Bound density $\rho_{\mathrm{b}}$ as a function of the spatial coordinate $x$ along the tube axis; (ii) Bound current $J_{\mathrm{b}}(x)$ which gives the number of motors that pass through a lattice site on the filament with coordinate $x$ per unit time; and (iii) Average bound current $\left\langle J_{\mathrm{b}}\right\rangle \equiv \int d x \rho_{\mathrm{b}}(x) / L$ which characterizes the overall transport along the filament.

In general, as one increases the total number of motors in the tube, or equivalently the concentration of these motors, the average bound current of motors on the filament increases first, but eventually reaches a maximal value and then starts to decrease as the traffic becomes jammed [17], see Fig. 4(a). The presence of stepping defects decreases the average bound current compared to the case without the defects for all choices of the total number of
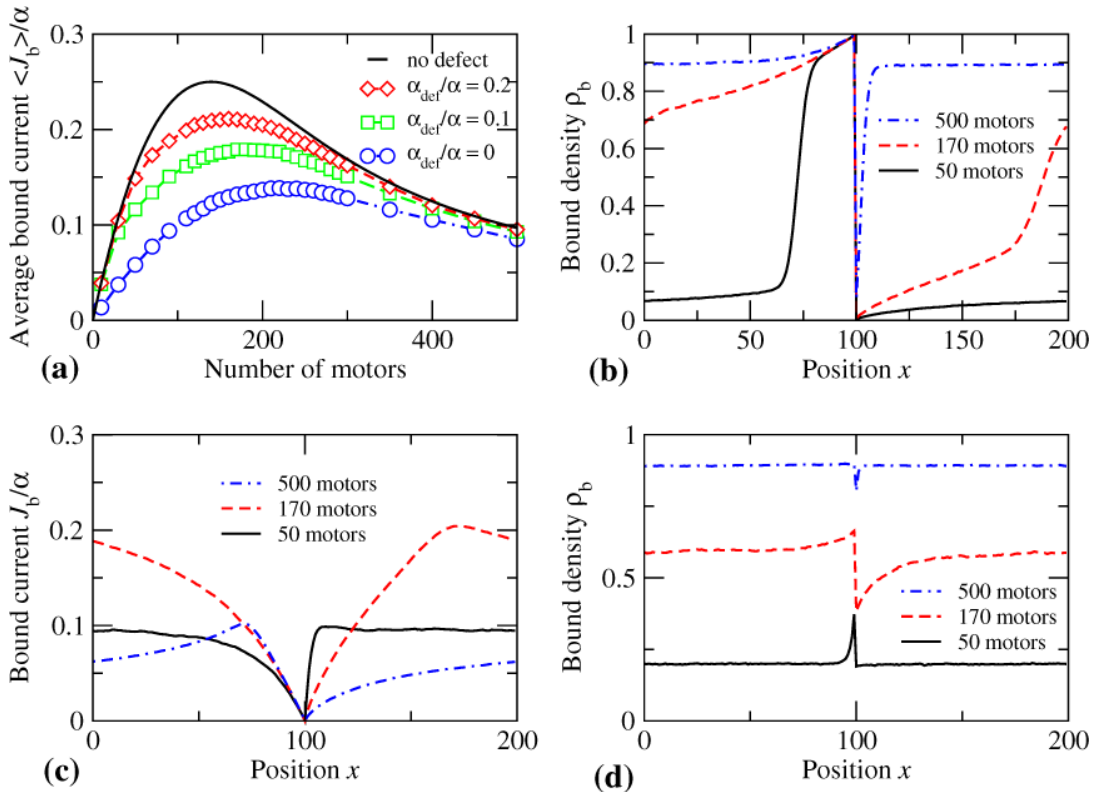

Fig. 4 Traffic of molecular motors in the presence of stepping defects: (a) Normalized average bound current $\left\langle J_{\mathrm{b}}\right\rangle / \alpha$ (in units of $\tau^{-1}$ ) as a function of the number of motors in the tube for different values of $\alpha_{\mathrm{def}}$. The black curve which gives the exact value of average bound current for the case without defects is calculated as in [17]. (b) Bound motor density $\rho_{\mathrm{b}}$ as a function of the coordinate $x$ (in units of $\ell$ ) along the tube for $\alpha_{\mathrm{def}}=0$ and different numbers of motors in the tube. (c) Corresponding normalized bound motor current $J_{\mathrm{b}} / \alpha$ (in units of $\tau^{-1}$ ) as a function of $x$. (d) Bound density profiles $\rho_{\mathrm{b}}(x)$ for a weaker stepping defect with $\alpha_{\mathrm{def}}=0.5 \alpha$. The parameters are the same as in Fig. 3 except for $L=200 \ell$ or, equivalently, $\rho_{\mathrm{def}}=0.005$ 
motors within the tube. The stronger the defects, the lower is the value of the average bound current. In addition, the curve for the average bound current as a function of the overall motor number becomes broader as the strength of the defects increases, and the maximum of the average bound current is shifted towards larger values of the overall motor number, see Fig. 4(a).

Density profiles of bound motors along the filament in the presence of stepping defects are shown in Fig. 4(b) for the limiting case $\alpha_{\text {def }}=0$, for which motors can only pass the defects by unbinding, diffusion and rebinding to the filament. These profiles show that stepping defects induce local traffic jams in front of the defect and a depletion zone after it. These profiles are very similar to those found in earlier studies on closed and half-open tube systems $[22,31,35]$ with the defect playing the role of the boundary of the tube. The spatial extension of the jammed region increases with the overall motor concentration. The end of the jammed region distal to the defect is marked by a rather sharp shock, i.e. a sudden change in density. The corresponding profiles of the current of bound motors are shown in Fig. 4(c). Weaker stepping defects with $\alpha_{\text {def }}>0$ cause a smaller perturbation of the bound density and bound current profiles; as shown in Fig. 4(d) for the case $\alpha_{\text {def }}=0.5 \alpha$, the effect of the defects is then confined to a small region around the defect.

\section{Transport by Molecular Motors in the Presence of Unbinding Defects}

\subsection{Single Motor with Unbinding Defects}

The second type of defects that we investigate is provided by unbinding defects. Motors at an unbinding defect site move forward with probability $\alpha$, unbind with probability $\epsilon_{\text {def }} / 6$ to each neighboring non-filament site and remain at the same position with probability $\gamma_{\text {def }}=$ $1-\alpha-4 \epsilon_{\text {def }} / 6$. We study again the case where the defects are regularly distributed on the filament with concentration $\rho_{\mathrm{def}}=\ell / L$ and we start by considering the effect of the defects on the movement of a single motor.

Since all sites have the same stepping parameter $\alpha$, the velocity of a bound motor, $v_{\mathrm{b}}=$ $\alpha \ell / \tau$ is not affected by the unbinding defects and the effective velocity, which is averaged over the bound and unbound states of the motor, is given by

$$
v_{\text {eff }}=\alpha P_{\mathrm{b}} \frac{\ell}{\tau}=\alpha\left(\sum_{x} \rho_{\mathrm{b}}(x)\right) \frac{\ell}{\tau} .
$$

Since the unbinding defects break the translational invariance of the system, they lead to inhomogeneous bound and unbound density profiles, so that the binding-unbinding balance is again not valid locally. As in the case of stepping defects, the bound and unbound motor densities satisfy a global balance of binding and unbinding

$$
\pi_{\mathrm{ad}} \sum_{x} \rho_{\mathrm{ub}}\left(x, y_{\mathrm{nn}}, z_{\mathrm{nn}}\right)=\sum_{x} \epsilon(x) \rho_{\mathrm{b}}(x),
$$

where $y_{\mathrm{nn}}$ and $z_{\mathrm{nn}}$ are again the perpendicular coordinates of a single channel of non-filament sites that are the nearest neighbors of the filament sites.

As a global property of motor unbinding, we introduce an effective unbinding probability, $\epsilon_{\text {eff, }}$ which is defined via

$$
\sum_{x} \epsilon(x) \rho_{\mathrm{b}}(x) \equiv \epsilon_{\mathrm{eff}} \sum_{x} \rho_{\mathrm{b}}(x)=\epsilon_{\mathrm{eff}} P_{\mathrm{b}}
$$


Using this relation in (17) together with the replacement of $\rho_{\mathrm{ub}}\left(x, y_{\mathrm{nn}}, z_{\mathrm{nn}}\right)$ by $\rho_{\mathrm{ub}}(x)$ and the normalization condition (7), the probability $P_{\mathrm{b}}$ as defined by (6) is now given by

$$
P_{\mathrm{b}}=\frac{\pi_{\mathrm{ad}}}{\pi_{\mathrm{ad}}+\epsilon_{\mathrm{eff}} N_{\mathrm{ch}}}
$$

instead of relation (9) for stepping defects. Furthermore, the flux balance relation (17) is equivalent to

$$
\pi_{\mathrm{ad}} \frac{P_{\mathrm{ub}}}{N_{\mathrm{ch}}}=\pi_{\mathrm{ad}} \frac{1-P_{\mathrm{b}}}{N_{\mathrm{ch}}}=\epsilon_{\mathrm{eff}} P_{\mathrm{b}} .
$$

It follows from (16) and (19) which replaces the relation (9) for stepping defects that the effective unbinding parameters $\epsilon_{\text {eff }}$ and the effective velocity $v_{\text {eff }}$ satisfy the relation:

$$
\epsilon_{\mathrm{eff}}=\frac{\pi_{\mathrm{ad}}}{N_{\mathrm{ch}}}\left(\frac{\alpha}{v_{\mathrm{eff}}} \frac{\ell}{\tau}-1\right)
$$

So far, we have only rewritten the motor properties in terms of the new parameter $\epsilon_{\text {eff }}$. In the following, we will consider several analytical approximations to determine the effective unbinding parameter $\epsilon_{\text {eff }}$, which then leads to estimates for the bound state probability $P_{\mathrm{b}}$ and the effective velocity $v_{\text {eff }}$.

The simplest ansatz for $\epsilon_{\text {eff }}$ is to take the average of the unbinding probability along the filament, which leads to

$$
\epsilon_{\mathrm{eff}} \simeq\langle\epsilon(x)\rangle=\epsilon+\frac{\ell}{L}\left(\epsilon_{\mathrm{def}}-\epsilon\right)=\epsilon+\rho_{\mathrm{def}}\left(\epsilon_{\mathrm{def}}-\epsilon\right),
$$

with the defect density $\rho_{\mathrm{def}}=\ell / L$. This approximation is valid if the bound motor density along the filament is approximately constant. The latter condition is fulfilled if the motor is fast with $\alpha \gg \epsilon$ and $\alpha \gg \epsilon_{\text {def }}$.

In the opposite limit of small stepping parameter $\alpha$, the flux balance arising from binding and unbinding events is approximately valid locally, i.e.

$$
\pi_{\mathrm{ad}} \rho_{\mathrm{ub}}(x) \approx \epsilon(x) \rho_{\mathrm{b}}(x)
$$

for small $\alpha$. This relation is exact in the equilibrium case with $\alpha=0$. Furthermore, for small $\alpha$, the unbound density varies very little and can be approximated by a constant $\rho_{\mathrm{ub}}$, which again becomes exact for the equilibrium case with $\alpha=0$. It then follows from (23) that the bound density $\rho_{\mathrm{b}}$ behaves as

$$
\rho_{\mathrm{b}}(x) \simeq \frac{\pi_{\mathrm{ad}} \rho_{\mathrm{ub}}}{\epsilon(x)},
$$

for small stepping probability $\alpha$. The probability $P_{\mathrm{ub}}$ for an unbound motor state is now given by

$$
P_{\mathrm{ub}}=N_{\mathrm{ch}} \sum_{x} \rho_{\mathrm{ub}}(x) \approx N_{\mathrm{ch}} \frac{L}{\ell} \rho_{\mathrm{ub}}
$$

and the probability for a bound motor state by

$$
P_{\mathrm{b}}=\sum_{x} \rho_{\mathrm{b}}(x) \approx \sum_{x} \frac{\pi_{\mathrm{ad}} \rho_{\mathrm{ub}}}{\epsilon(x)} .
$$


Inserting the two expressions (25) and (26) into (20), one obtains the relation

$$
\frac{1}{\epsilon_{\mathrm{eff}}} \approx \frac{\ell}{L} \sum_{x=\ell}^{L} \frac{1}{\epsilon(x)}=\frac{1}{\epsilon}+\frac{\ell}{L}\left(\frac{1}{\epsilon_{\mathrm{def}}}-\frac{1}{\epsilon}\right) .
$$

for the effective unbinding probability $\epsilon_{\text {eff }}$ in the limit of small stepping parameter $\alpha$. Note that in this limit of small $\alpha$, one has to average the inverse of the local unbinding parameter rather than the unbinding parameter itself as in the limit of large $\alpha$. Expanding the relation (27) in powers of the defect density $\rho_{\text {def }}=\ell / L$ leads to

$$
\epsilon_{\mathrm{eff}} \approx \epsilon+\frac{\ell}{L}\left(\epsilon_{\mathrm{def}}-\epsilon\right) \frac{\epsilon}{\epsilon_{\mathrm{def}}} .
$$

Comparison of this result for small $\alpha$ with (22), which is valid for large $\alpha$, shows that in both cases $\left(\epsilon_{\text {eff }}-\epsilon\right) \sim \ell / L=\rho_{\text {def }}$, but with different prefactors. These relations are confirmed by simulations, see Fig. 5(a). Furthermore, the simulation data show how intermediate values of $\alpha$ interpolate between these limiting cases. For these intermediate values, which are typical for motors with finite processitivity, the effective unbinding probability exhibits a weak dependence on $\alpha$. The simulation data are described rather well by the expression

$$
\epsilon_{\mathrm{eff}}=\epsilon+\rho_{\mathrm{def}}\left(\epsilon_{\mathrm{def}}-\epsilon\right) \frac{\epsilon+q^{\prime} \alpha}{\epsilon_{\mathrm{def}}+q^{\prime} \alpha},
$$

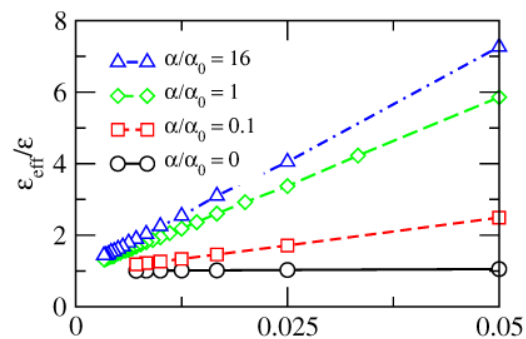

(a) Density of unbinding defects $\rho_{\text {def }}$

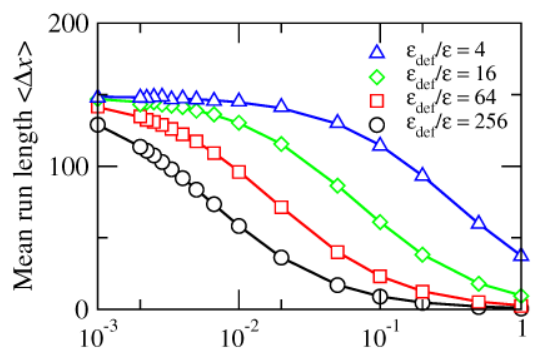

(c) Density of unbinding defects $\rho_{\text {def }}$

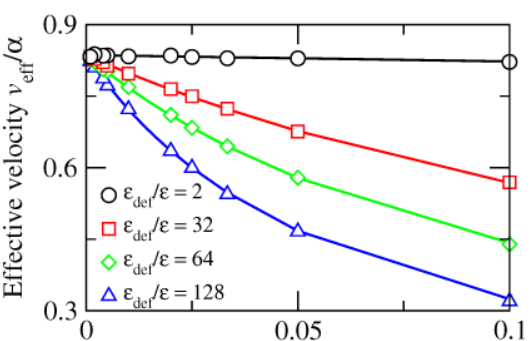

(b) Density of unbinding defects $\rho_{\text {def }}$

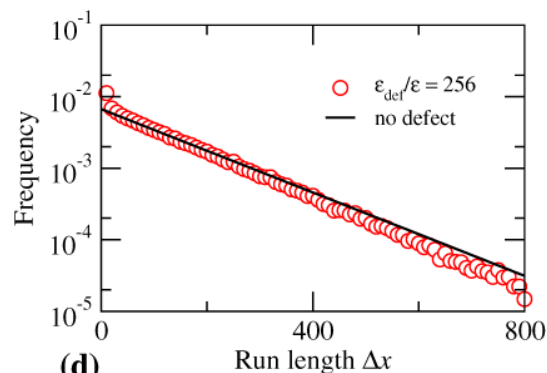

(d)

Fig. 5 Transport properties of single motors in the presence of unbinding defects: (a) Normalized effective unbinding probability $\epsilon_{\text {eff }} / \epsilon$ obtained from simulations via (21). (b) Normalized effective velocity $v_{\text {eff }} / \alpha$ (in units of $\ell / \tau$ ). (c) Average run length $\langle\Delta x\rangle$ (in units of $\ell$ ) as a function of the defect density $\rho_{\text {def. The lines in }}$ (b) and (c) are fits to the simulation data using (29) combined with (21) and (30), respectively. (d) Run length distribution for different defect strengths $\epsilon_{\mathrm{def}} / \epsilon$. The straight line corresponds to the exponential function $(4 \epsilon / 6 \alpha \ell) \exp (-4 \Delta x \epsilon / 6 \alpha \ell)$ of run length distribution without defects. The parameters are the same as in Fig. 3 except for $\alpha_{0}=0.0099333$ in (a) and for $N_{\mathrm{ch}}=316$ and $L / \ell=1000$ in (d) 
where $q^{\prime}$ is a free parameter determined to be $q^{\prime} \simeq 4.0$ by fitting the simulation data. Note that the expression (29) interpolates between (22) for large $\alpha$ and (28) for small $\alpha$. Using (29) also leads to a rather accurate description of the motor velocity as obtained from simulations, see Fig. 5(b), where the motor velocity is shown as a function of defect density and defect strength, i.e. the defect unbinding probability. We note that small defect densities can have a rather strong effect, if the unbinding probability at the defect is of the same order of magnitude as the stepping probability $\alpha$. For example in the case $\epsilon_{\text {def }}=128 \epsilon$, a defect density of about 4 percent reduces the effective velocity two-fold and a 10 percent defect density reduces it three-fold.

Unbinding defects also reduce the run length of the motor. In the presence of unbinding defects, the mean run length can be expressed in terms of the effective unbinding probability as

$$
\langle\Delta x\rangle=\frac{\alpha}{4 \epsilon_{\mathrm{eff}} / 6} \ell,
$$

as obtained from (14) when $\epsilon$ is replaced by $\epsilon_{\text {eff }}$. The dependence of the mean run length on the defect strength and the defect density is shown in Fig. 5(c). As in the case of stepping defects studied above, see Fig. 3, small defect densities have only a weak effect on the run length, while large defect densities shorten the runs strongly. The crossover density at which the effect of the defects becomes notable has a strong dependence on the defects strength, i.e. $\epsilon_{\mathrm{def}}$, and can be quite small for strong defects with large $\epsilon_{\mathrm{def}}$. For example, for $\epsilon_{\mathrm{def}}=256 \epsilon$, a two-fold reduction of the mean run length is obtained for $\rho_{\text {def }} \simeq 0.007$, that is if less than one percent of the filament sites are unbinding defects. The effect of unbinding defects on the run length is very similar to the corresponding effect of stepping defects, see Fig. 3. This similarity reflects the fact that the effects of strong stepping and unbinding defects have some common aspects: when the motor encounters the defects, it has a rather high probability to unbind from the filament, either because of the high unbinding probability at an unbinding defect or because of the prolonged sojourn time at a stepping defect.

As a consequence, unbinding and stepping defects also have a similar effect on the run length distributions, compare Fig. 5(d) and 3(c). Figure 5(d) shows run length distributions for a rather low density of unbinding defects. As in the case of stepping defects, the length scale that governs the exponential decay of the distribution is slightly reduced, and the distributions exhibit a peak at small run lengths.

\subsection{Many Motors with Unbinding Defects}

Now let us consider the effect of unbinding defects on the traffic of many motors which interact through mutual exclusion. Figure 6(a) shows the average bound current as a function of the overall number of motors within the tube for a low defect density. It can be seen from these plots that the binding defects do not always reduce the current, as one might expect and as found for the stepping defects discussed above, see Fig. 4(a). For small motor numbers, the average bound current is indeed slightly reduced by the presence of the defects, but for large motor numbers, the current is slightly increased. This observation can be understood as follows: For small motor concentration, the decrease of the bound motor density arising from the unbinding defects leads to a reduction of the average bound current. If the concentration of motors is however larger than the concentration for which the average bound current attains its maximal value, a reduction of the bound motor density leads to an increase of the average bound current, because the increased unbinding probability relieves the traffic jams appearing for high motor densities. 


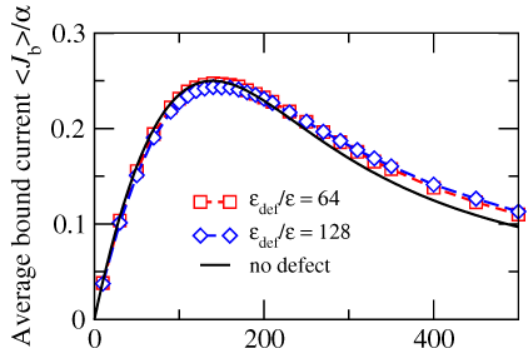

(a)

Number of motors

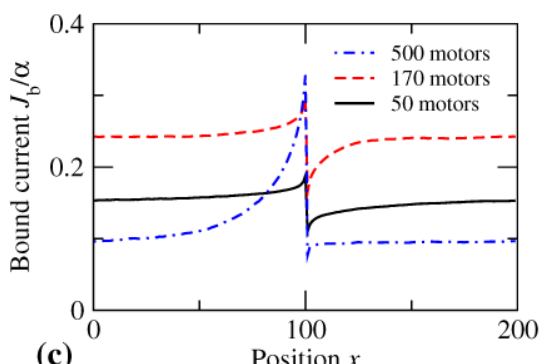

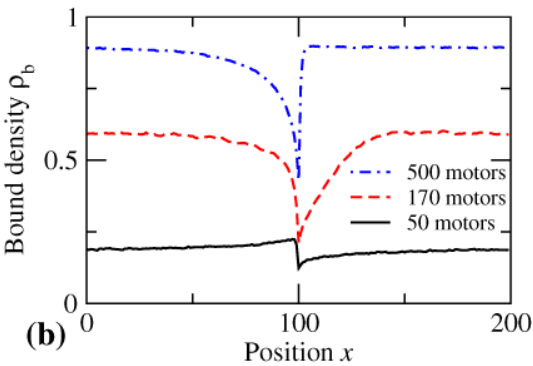

(b)

Position $x$

Fig. 6 Motor traffic in the presence of unbinding defects: (a) Normalized average bound current $\left\langle J_{\mathrm{b}}\right\rangle / \alpha$ (in units of $\tau^{-1}$ ) as a function of the number of motors for different values of $\epsilon_{\mathrm{def}}$. The black curve which gives the exact value of average bound current for the case without defects is calculated as in [17]. (b) Bound motor density $\rho_{\mathrm{b}}$ as a function of the spatial coordinate $x$ (in units of $\ell$ ) along the tube axis. (c) Corresponding profiles of the bound motor current $J_{\mathrm{b}}(x)$ (in units of $\tau^{-1}$ ). The parameters are the same as in Fig. 4, with $\epsilon_{\mathrm{def}}=128 \epsilon$ in (b) and (c)

Profiles of the bound motor densities on the filament are shown in Fig. 6(b). The profiles are rather constant away from the defect, but have a minimum at the defect site. This is what one would expect, since motors unbind from the filament at this site. For small overall motor concentration (or total motor number), the profiles also exhibit a maximum in front of the defect. This maximum arises from the locally increased density of unbound motors which leads to increased rebinding of motors to the filament. Since this maximum requires a locally increased motor density, it is only present if the diffusion of unbound motors is not too fast. When the overall motor concentration is increased, this maximum disappears. The corresponding bound current profiles are shown in Fig. 6(c) for a strong defect with $\epsilon_{\text {def }}=128 \epsilon$. The current exhibits a peak in front of the defects and a depletion zone behind these defects. The depletion zone behind the defect follows the bound density profile closely, which indicates that it reflects the reduced motor density due to unbinding at the defect. On the other hand, the peak in front of the defect is present when the bound density exhibits a peak, as well as for high motor concentration when no density peak occurs.

\section{Transport by Molecular Motors in the Presence of Binding Defects}

\subsection{Single Motor with Binding Defects}

The third class of defects we investigate are binding defects. This type of defect appears to be the most common one in biological systems as shown in Table 1. At a binding defect site, 


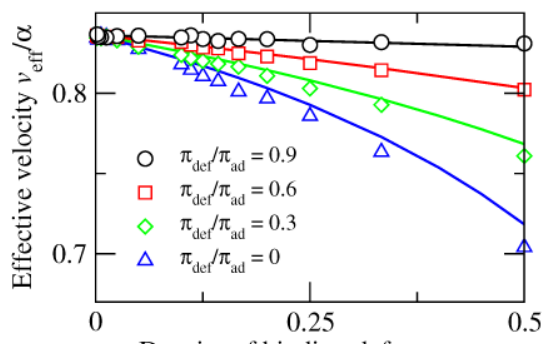

(a) Density of binding defects $\rho_{\text {def }}$

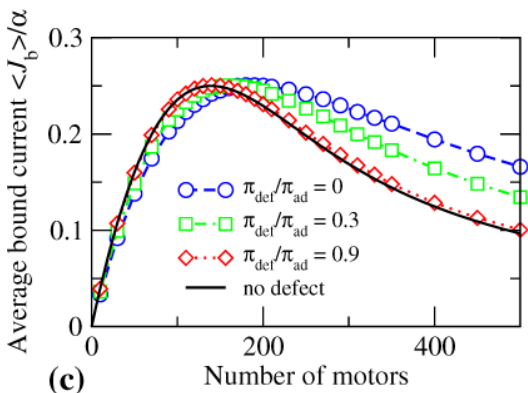

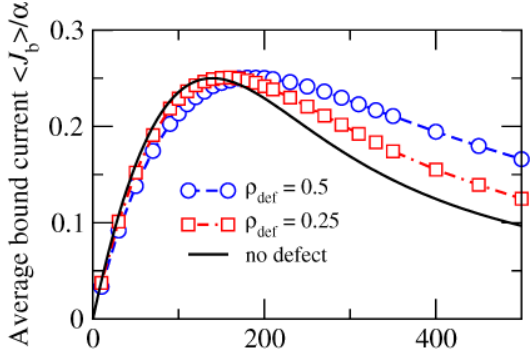

(b) Number of motors

Fig. 7 Transport properties of single motors and motor traffic in the presence of binding defects: (a) Normalized velocity $v_{\text {eff }} / \alpha$ of a single motor as a function of the density $\rho_{\text {def }}$ of binding defects for different values of $\pi_{\mathrm{def}}$. The symbols show simulation data and the lines the corresponding results from a mean-field calculation. (b) Normalized average bound current $\left\langle J_{\mathrm{b}}\right\rangle / \alpha$ (in units of $\tau^{-1}$ ) as a function of the number of motors in the tube for $\pi_{\text {def }}=0$ and different defect densities $\rho_{\text {def }}$. (c) Motor current (in units of $\tau^{-1}$ ) for $\rho_{\text {def }}=0.5$ and different defects strengths $\pi_{\text {def }}$. The black curves in (b) and (c) which give the exact value of average bound current for the case without defects are calculated as in [17]. The other parameters are the same as in Fig. 4

bound motors have the same hopping probabilities as at any other filament site, but unbound motors that approach the binding defect site have a reduced sticking probability $\pi_{\mathrm{def}}$.

Similar to the case of unbinding defects as studied in the previous section, binding defects do not affect the movement of bound motors, but rather change the balance of binding and unbinding. The stronger the binding defects are, or the higher the density of binding defects on the filament is, the less likely it becomes for motors to bind to the filament. A local balance of binding and unbinding, similar to (17), is also valid in this case, but now with a site-dependent binding probability.

Simulation results for the effective motor velocity as a function of the density of binding defects are shown in Fig. 7(a). This figure also includes results obtained from a mean field approximation using an effective (site-independent) binding parameter $\pi_{\mathrm{eff}} \simeq\left\langle\pi_{\mathrm{ad}}(x)\right\rangle=$ $\pi_{\mathrm{ad}}+\rho_{\mathrm{def}}\left(\pi_{\mathrm{def}}-\pi_{\mathrm{ad}}\right)$. As can be seen in Fig. 7(a), the latter approximation leads to good agreement with the simulation data. The most noticeable feature of Fig. 7(a) is that the effect of binding defects is rather weak. Binding defects only have a notable effect at high defect densities. But even if binding is completely suppressed at every second filament site, i.e. for $\rho_{\mathrm{def}}=0.5$ and $\pi_{\mathrm{def}}=0$, the effect remains weak, since the effective motor velocity is only decreased by 14 percent. This result is in striking contrast to the strong effects of the other two defects types.

As the binding defects do not affect the movement of bound motors, the run length is not changed compared to the case without defects. 


\subsection{Many Motors with Binding Defects}

Finally we investigate the traffic of many motors in the presence of binding defects. Figure 7(b) shows the average bound current as a function of the number of motors in the tube for binding defects with $\pi_{\text {def }}=0$. As in the case of unbinding defects shown in Fig. 6(a), binding defects can both increase and decrease the bound motor current. For low motor concentrations, binding defects reduce the current by reducing the probability that a motor is bound. For large motor concentrations, the binding defects increase the current compared to the case without defects, because the binding defects reduce the motor traffic jams on the filament. For weaker binding defects with $\pi_{\text {def }}>0$, the effect is similar, but even smaller. The maximal value of the average bound current is not changed by the presence of binding defects, the defects rather shift the current maximum to larger motor numbers, see Figs. 7(b) and 7(c). Again, the effect of binding defects is much weaker than that of stepping or unbinding defects. Binding defects only have a notable effect on the traffic of many motors when the defect density is sufficiently large.

\subsection{Binding Defects and Cooperative Transport by Several Motors}

We have noted above that binding defects account for most of the biologically relevant defects. In striking contrast to their importance in biological systems, our analysis shows that binding defects have very small effects on both the movements of individual motors and on the traffic of many motors. It is important to note, however, that our conclusions about binding defects apply only to the traffic of individual motor molecules or to the traffic of cargo particles that are pulled by single motors. Unbinding defects are expected to have a much stronger effect on cargo particles that are pulled by teams of several motors, the typical situation for in vivo transport $[1,21,36]$. Thus, let us consider a cargo particle that is pulled by $N$ identical motors such as kinesin. The effective unbinding rate $\bar{\epsilon}_{\text {eff }}$ of such a cargo particle is proportional to $\left(\epsilon / \pi_{\mathrm{ad}}\right)^{N-1}$ for strongly binding motors with $\epsilon / \pi_{\mathrm{ad}} \ll 1$ [21] where $\epsilon$ and $\pi_{\mathrm{ad}}$ are the previously defined unbinding and sticking probabilities of a single motor. Thus, for a cargo particle pulled by $N$ strongly binding motors, the effective unbinding rate $\bar{\epsilon}_{\text {eff }} \sim\left(1 / \pi_{\mathrm{ad}}\right)^{N-1}$ and is, thus, strongly affected by the value $\pi_{\text {ad }}$ for the sticking probability of a single motor. This implies that binding defects for single motors will act as unbinding defects for cargo particle pulled by several motors. This effect has indeed been demonstrated experimentally for tau proteins: As expected for binding defects, tau proteins do not affect the run length of individual motors $[48,52]$. However, tau proteins strongly reduce the run length for cargoes pulled by several motors [52] and, thus, act as effective unbinding defects. A quantitative description for this latter effect can be obtained by an extension of the models studied here and in [21,36].

\section{Summary}

In this article, we have studied the traffic of molecular motors in the presence of different types of static defects on the filament. We have determined several properties that characterize the movement of single motors as well as the traffic behavior in many-motor systems such as motor velocities, motor run length, motor density and current profiles.

We have considered three basic types of static defects, namely stepping, unbinding and binding defects. At the defect sites, the dynamics of the motors differs in only one transition probability from the dynamics at other filament sites. While stepping defects and unbinding 
defects have rather strong effects on the motor behavior and severely reduce the velocity, run length and currents, the effect of binding defects on individual motors is much weaker and becomes only notable if the density of the defects is sufficiently large. The run length is not affected at all by binding defects. At first sight, these results appear to be at odds with the experimental observation that most biologically relevant defects such as microtubuleassociated proteins (MAPs) represent binding defects as summarized in Table 1. It is, however, plausible that MAPs mainly regulate the movement of larger cargoes, which are pulled by several motors. For such cargo particles, the effective unbinding rate $\bar{\epsilon}_{\text {eff }}$ depends rather strongly on the binding probability $\pi_{\mathrm{ad}}$ of individual motors as discussed in Sect. 5.3. Thus, in order to describe the regulation of cargo particles by, e.g., MAPs, one should extend the models discussed here to cooperative transport by teams of motors.

In general, localized inhomogeneities on filaments, which may be both modifications of the filament itself or other molecules bound to the filaments, can modulate the patterns of molecular motor transport in various ways. While most of the systems we considered reduce motor movements, we note that unbinding defects can increase the motor current if the local motor density is high. In addition, it is easy to imagine binding defects that increase motor binding and function as loading stations that initiate filament transport, although we are not aware of any biological system with this function. In addition to their functions for intracellular transport, regulatory mechanisms via filament defects or inhomogeneities may also be of interest for the development of artificial biomimetic transport systems based on molecular motors $[13,51]$.

Acknowledgements The authors thank Melanie Müller for fruitful discussions. SK was supported by Deutsche Forschungsgemeinschaft (Grants KL818/1-1 and 1-2) and by the NSF through the Center for Theoretical Biological Physics (Grant PHY-0822283).

Open Access This article is distributed under the terms of the Creative Commons Attribution Noncommercial License which permits any noncommercial use, distribution, and reproduction in any medium, provided the original author(s) and source are credited.

\section{References}

1. Beeg, J., Klumpp, S., Dimova, R., Gracia, R.S., Unger, E., Lipowsky, R.: Transport of beads by several kinesin motors. Biophys. J. 94, 532-541 (2008)

2. Bulisnki, J.C., McGraw, T.E., Gruber, D., Nguyen, H.L., Sheetz, M.P.: Overexpression of MAP4 inhibits organelle motility and trafficking in vivo. J. Cell Sci. 110, 3055-3064 (1997)

3. Crevel, I.M., Nyitrai, M., Alonso, M.C., Weiss, S., Geeves, M.A., Cross, R.A.: What kinesin does at roadblocks: The coordination mechanism for molecular walking. Eur. Mol. Biol. Organ. J. 23, 23-32 (2004)

4. Davis, L.J., Odde, D.J., Block, S.M., Gross, S.P.: The importance of lattice defects in katanin-mediated microtubule severing in vitro. Biophys. J. 82, 2916-2927 (2002)

5. Dixit, R., Ross, J.L., Goldman, Y.E., Holzbaur, E.L.F.: Differential regulation of dynein and kinesin motor proteins by tau. Science 319, 1086-1089 (2008)

6. Dreblow, K., Kalchishkova, N., Böhm, K.J.: Kinesin bypassing blockages on microtubule rails. Biophys. Rev. Lett. (in press)

7. Ebneth, A., Godemann, R., Stamer, K., Illenberger, S., Trinczek, B., Mandelkow, E.-M., Mandelkow, E.: Overexpression of tau protein inhibits kinesin-dependent trafficking of vesicles, mitochondria, and endoplasmic reticulum: Implications for Alzheimer's disease. J. Cell Biol. 143, 777-794 (1998)

8. Evans, M.R., Juhász, R., Santen, L.: Shock formation in an exclusion process with creation and annihilation. Phys. Rev. E 68, 026117 (2003)

9. Goldstein, L.S.B., Yang, Z.: Microtubule-based transport systems in neurons: The roles of kinesins and dyneins. Annu. Rev. Neurosci. 23, 39-71 (2000)

10. Grzeschik, H., Harris, R.J., Santen, L.: Traffic of cytoskeletal motors with disordered attachment rates. Preprint arXiv:0806.3845 (2008) 
11. Hagiwara, H., Yorifuji, H., Sato-Yoshitake, R., Hirokawa, N.: Competition between motor molecules (kinesin and cytoplasmic dynein) and fibrous microtubule-associated proteins in binding to microtubules. J. Biol. Chem. 269, 3581-3589 (1994)

12. Heins, S., Song, Y.H., Wille, H., Mandelkow, E., Mandelkow, E.-M.: Effect of MAP2, MAP2c, and tau on kinesin-dependent microtubule motility. J. Cell Sci. Suppl. 14, 121-124 (1991)

13. Hess, H., Bachand, G.D., Vogel, V.: Powering nanodevices with biomolecular motors. Chem. Eur. J. 10, 2110-2116 (2004)

14. Howard, J.: Mechanics of Motor Proteins and the Cytoskeleton. Sinauer Associates, Sunderland (2001)

15. Janowsky, S.A., Lebowitz, J.L.: Finite-size effects and shock fluctuations in the asymmetric simpleexclusion process. Phys. Rev. A 45, 618-625 (1992)

16. Klein, G.A., Kruse, K., Cuniberti, G., Jülicher, F.: Filament depolymerization by motor molecules. Phys. Rev. Lett. 94, 108102 (2005)

17. Klumpp, S., Lipowsky, R.: Traffic of molecular motors through tube-like compartments. J. Stat. Phys. 113, 233-268 (2003)

18. Klumpp, S., Lipowsky, R.: Asymmetric simple exclusion processes with diffusive bottlenecks. Phys. Rev. E 70, 066104 (2004)

19. Klumpp, S., Lipowsky, R.: Phase transitions in systems with two species of molecular motors. Europhys. Lett. 66, 90-96 (2004)

20. Klumpp, S., Lipowsky, R.: Active diffusion of motor particles. Phys. Rev. Lett. 95, 268102 (2005)

21. Klumpp, S., Lipowsky, R.: Cooperative cargo transport by several molecular motors. Proc. Natl. Acad. Sci. USA 102, 17284-17289 (2005)

22. Klumpp, S., Nieuwenhuizen, T.M., Lipowsky, R.: Self-organized density patterns of molecular motors in arrays of cytoskeletal filaments. Biophys. J. 88, 3118-3132 (2005)

23. Klumpp, S., Chai, Y., Lipowsky, R.: Effects of the chemomechanical stepping cycle on the traffic of molecular motors. Phys. Rev. E 78, 041909 (2008)

24. Kolomeisky, A.B.: Asymmetric simple exclusion model with local inhomogeneity. J. Phys. A Math. Gen. 31, 1153-1164 (1998)

25. Konzack, S., Rischitor, P.E., Enke, C., Fischer, R.: The role of the kinesin motor KipA in microtubule organization and polarized growth of Aspergillus nidulans. Mol. Biol. Cell 16, 497-506 (2005)

26. Krug, J.: Phase separation in disordered exclusion models. Braz. J. Phys. 30, 97-104 (2000)

27. Lakämper, S., Meyhöfer, E.: The E-hook of tubulin interacts with kinesin's head to increase processivity and speed. Biophys. J. 89, 3223-3234 (2005)

28. Larcher, J.-C., Boucher, D., Lazereg, S., Gros, F., Denoulet, P.: Interaction of kinesin motor domains with $\alpha$ - and $\beta$-tubulin subunits at a tau-independent binding site: Regulation by polyglutamylation. J. Biol. Chem. 271, 22117-22124 (1996)

29. Leduc, C., Campàs, O., Zeldovich, K.B., Roux, A., Jolimaitre, P., Bourel-Bonnet, L., Goud, B., Joanny, J.-F., Bassereau, P., Prost, J.: Cooperative extraction of membrane nanotubes by molecular motors. Proc. Natl. Acad. Sci. USA 101, 17096-17101 (2004)

30. Lipowsky, R., Klumpp, S.: 'Life is motion': Multiscale motility of molecular motors. Physica A 352, 53-112 (2005)

31. Lipowsky, R., Klumpp, S., Nieuwenhuizen, T.M.: Random walks of cytoskeletal motors in open and closed compartments. Phys. Rev. Lett. 87, 108101 (2001)

32. Lopez, L.A., Sheetz, M.P.: Steric inhibition of cytoplasmic dynein and kinesin motility by MAP2. Cell Motil. Cytoskelet. 24, 1-16 (1993)

33. Mandelkow, E.-M., Thies, E., Trinczek, B., Biernat, J., Mandelkow, E.: MARK/PAR1 kinase is a regulator of microtubule-dependent transport in axons. J. Cell Biol. 167, 99-110 (2004)

34. Mirin, N., Kolomeisky, A.B.: Effect of detachments in asymmetric simple exclusion processes. J. Stat. Phys. 110, 811-823 (2003)

35. Müller, M.J.I., Klumpp, S., Lipowsky, R.: Molecular motor traffic in a half-open tube. J. Phys. Condens. Matter 17, S3839-S3850 (2005)

36. Müller, M.J.I., Klumpp, S., Lipowsky, R.: Tug-of-war as a cooperative mechanism for bidirectional cargo transport by molecular motors. Proc. Natl. Acad. Sci. USA 105, 4609-4614 (2008)

37. Nieuwenhuizen, T.M., Klumpp, S., Lipowsky, R.: Random walks of molecular motors arising from diffusional encounters with immobilized filaments. Phys. Rev. E 69, 061911 (2004)

38. Nishinari, K., Okada, Y., Schadschneider, A., Chowdhury, D.: Intracellular transport of single-headed molecular motors KIF1A. Phys. Rev. Lett. 95, 118101 (2005)

39. Parmeggiani, A., Franosch, T., Frey, E.: Phase coexistence in driven one-dimensional transport. Phys. Rev. Lett. 90, 086601 (2003)

40. Paschal, B.M., Obar, R.A., Vallee, R.B.: Interaction of brain cytoplasmic dynein and MAP2 with a common sequence at the $\mathrm{C}$ terminus of tubulin. Nature 342, 569-572 (1989) 
41. Pierobon, P., Mobilia, M., Kouyos, R., Frey, E.: Bottleneck-induced transitions in a minimal model for intracellular transport. Phys. Rev. E 74, 031906 (2006)

42. Reed, N.A., Cai, D., Blasius, T.L., Jih, G.T., Meyhofer, E., Gaertig, J., Verhey, K.J.: Microtubule acetylation promotes kinesin-1 binding and transport. Curr. Biol. 16, 2166-2172 (2006)

43. Sato-Harada, R., Okabe, S., Umeyama, T., Kanai, Y., Hirokawa, N.: Microtubule-associated proteins regulate microtubule function as the track for intracellular membrane organelle transports. Cell Struct. Funct. 21, 283-295 (1996)

44. Schliwa, M. (ed.): Molecular Motors. Wiley/VCH, Weinheim (2003)

45. Schmittmann, B., Zia, R.K.P. Statistical mechanics of driven diffusive systems. In: Domb, C., Lebowitz, J. (eds.) Phase Transitions and Critical Phenomena, vol. 17. Academic Press, London/San Diego (1995)

46. Schütz, G.M.: Exactly solvable models for many-body systems far from equilibrium. In: Domb, C., Lebowitz, J. (eds.) Phase Transitions and Critical Phenomena, vol. 19, pp. 1-251. Academic Press, San Diego (2001)

47. Seitz, A., Surrey, T.: Processive movement of single kinesins on crowded microtubules visualized using quantum dots. Eur. Mol. Biol. Organ. J. 25, 267-277 (2006)

48. Seitz, A., Kojima, H., Oiwa, K., Mandelkow, E.-M., Song, Y.H., Mandelkow, E.: Single-molecule investigation of the interference between kinesin, tau and MAP2c. Eur. Mol. Biol. Organ. J. 21, 4896-4905 (2002)

49. Tokuraku, K., Noguchi, T.Q.P., Nishie, M., Matsushima, K., Kotani, S.: An isoform of microtubuleassociated protein 4 inhibits kinesin-driven microtubule gliding. J. Biochem. 141, 585-591 (2007)

50. Tripathy, G., Barma, M.: Steady state and dynamics of driven diffusive systems with quenched disorder. Phys. Rev. Lett. 78, 3039-3042 (1997)

51. Van den Heuvel, M.G.L., Dekker, C.: Motor proteins at work for nanotechnology. Science 317, 333-336 (2007)

52. Vershinin, M., Carter, B.C., Razafsky, D.S., King, S.J., Gross, S.P.: Multiple-motor based transport and its regulation by Tau. Proc. Natl. Acad. Sci. USA 104, 87-92 (2007)

53. Vershinin, M., Xu, J., Razafsky, D.S., King, S.J., Gross, S.P.: Tuning microtubule-based transport through filamentous MAPs: The problem of dynein. Traffic 9, 882-892 (2008) 